\title{
Mobile Healthcare System Based on Bluetooth Medical Device
}

\author{
Jeong-Heon Kim¹, Seung-Chul Lee², Boon-Giin Lee1, and Wan-Young Chung ${ }^{3,+}$
}

\begin{abstract}
Recently healthcare industry such as pharmaceutical, medical device and healthcare service technology is growing significantly. Mobile healthcare has attracted big attention due to IT convergence technology. Paradigm of healthcare has been changed from the 1st generation(communicable disease prevention) and the 2nd generation(treatment of disease due to extended life expectancy) to the 3rd generation(extended life expectancy due to prevention and control). In our study, we suggest the 3rd generation mobile healthcare system using Bluetooth based wearable ECG monitoring system and smart phone technology. The mobile healthcare system consists of wearable shirts with Bluetooth communication module, ECG sensor, battery, and mobile phone. The ECG data is obtained by a miniaturized sensor and the data is transferred to a mobile phone using Bluetooth communication. Then, user can monitor his/her own ECG signal on an application using Android in mobile phone. The Bluetooth communication device is used due to highly reliable data transmission property and the Bluetooth chip is embedded in every mobile phone. The wearable shirts with chest belt of Bluetooth ECG module is designed with a focus on convenience in the daily life of a wearer. The ECG signal evaluation software in Android based mobile phone is developed for the health check and the ECG signal variation is tested according to the activities of the wearer such as walking, climbing stairs, stand up and sit down, and so on.
\end{abstract}

Keywords : Mobile Healthcare, ECG, Bluetooth Communication, Smart Phone, Ubiquitous Computing

\section{INTRODUCTION}

Recently, patients with chronic diseases such as cancer, diabetes, hypertension or cardiovascular disease are increasing due to westernized eating habits and aging of population. Healthcare cost is increasing due to the increase in the number of patient. This causes the international competitiveness of medical and health services to be improved[1].

Providing patients with convenient health facilities at a lower cost has always been a great challenge for health service providers. Moreover, the fast changing lifestyle of the modern world and the problem of aging society pose an urgent need to modernize such facilities. This involves devising cheaper and smarter ways of providing healthcare to people suffering from age related disease.

The u-healthcare(ubiquitous healthcare) technology is a combination of information communication with medical service to allow a patient to take care of himself or to allow a caregiver to take care of the patient at anywhere and anytime. To provide much freedom to patients in the u-

${ }^{1}$ Department of Electronic Engineering, Graduate School, ${ }^{2}$ Korea Electronics Technology Institute

${ }^{3}$ Department of Electronic Engineering, Pukyong National University

${ }^{+}$Corresponding author: wychung@pknu.ac.kr

(Received : Apr. 17, 2012, Revised : Jun. 25, 2012, Accepted : Jul. 2, 2012) health service environment, it is important to integrate wireless communication technology for modern healthcare systems[2]. Several biomedical meters or sensors such as glucose meter, pulse oximeter, ECG sensor are used for the u-healthcare system. There are mainly two communication methods to provide freedom to patients who take biomedical sensor modules on the body. Wireless network and wireless sensor network(WSN) have been used for the u-healthcare services[3]. Until now Bluetooth or ZigBee communication module is used for the data transfer from sensor nodes attached on the patient's body to the gateway or cellular phone. The personal health data gathered at the gateway is sent to the server computer through the internet. ZigBee is an alliance, without sprit of lucre, of more than 100 companies, where most of them are semiconductor manufacturers. ZigBee and IEEE 802.15.4 are standardsbased protocols that provide the network infrastructure required for WSN applications. The 802.15.4 defines the physical and MAC(Media Access Control) layers, and ZigBee defines the network and application layers. This technology is focused on creating low-rate personal area networks(LR-WPAN). In wireless sensor network, the biomedical data is transferred by multi-hop communication via several wireless sensor nodes. It is presented as a very economical solution for WSN. ZigBee products work in a 
band of frequencies that includes $2.4 \mathrm{GHz}$, and $868 \mathrm{MHz}$ in Europe or $915 \mathrm{MHz}$ in North America. It consumes very low energy and battery can be kept for several years. On the contrary, their typical coverage is around $50 \mathrm{~m}$, although this value could increase, depending on the environment[4].

However, Bluetooth is open specification for wireless networks based on radiofrequency that operates in the industrial scientific medical frequency band $(2 \mathrm{GHz}, 4 \mathrm{GHz})$ forming wireless personal area networks(WPANs). Bluetooth controls its interference and susceptibility to interference by using spectrum modulation. Bluetooth was formalized in the IEEE 802.15.1 standard. In Bluetooth, 79 different channels can coexist, although there are no defined routing capacities for multi-hop wireless sensor network. This technology achieves $100 \mathrm{~m}$ of coverage radius, although it consumes higher energy than ZigBee communication and battery can last for several days. The one of advantage of Bluetooth technology over ZigBee in healthcare is smart phone compatibility. Recently all the smart phones have a built-in Bluetooth chip[5]. Therefore, every sensor with Bluetooth communication module attached on the body of a patient can transmit the biomedical data to the mobile phone[6].

This paper presents a mobile healthcare system for the continuous monitoring of patients under their natural physiological states or elderly persons with chronic diseases. Our system is designed specifically for the patients who wear wearable shirts with imbedded ECG sensor with a focus on convenience in daily life of wearer. A small size ECG sensor with Bluetooth communication module is designed and fabricated. The ECG signal evaluation software in Android based smart phone is developed for the health check and the ECG signal variation is tested according to the activities of the wearer such as walking, climbing stairs, stand up and sit down, and so on.

\section{SYSTEM CONFIGURATION}

A mobile healthcare(m-healthcare) system which monitors health status and daily life activities of patient or elderly by using wearable ECG sensor alone attached on the body of the wearer is proposed. The m-healthcare allows seamless data analysis and diagnosis. Until now two kinds of mobile healthcare system have been developed. The first one sends the health data from the cellular phone to the internet connected to the server PC. Then, a doctor or caregiver checks the health status of the patient or elderly through internet. The mobile healthcare system is connected continuously to the internet and has to pay expensive data fee. The second one monitors the health status of cellular phone holder by the evaluation and monitoring software of the biomedical signal in the cellular phone. Our mobile healthcare system is the second one and for the patient, elderly or normal person itself who has cellular phone, smart phone or tablet PC such as Galaxytab(Samsung Electronics Co. Ltd., Korea) and ipad(Apple Inc., USA). Fig. 1 shows the proposed architecture of $\mathrm{m}$-healthcare system. The proposed system consists of hardware and software. Hardware consists of ECG module, MCU, smart monitoring device and software consists of application based on Android OS.

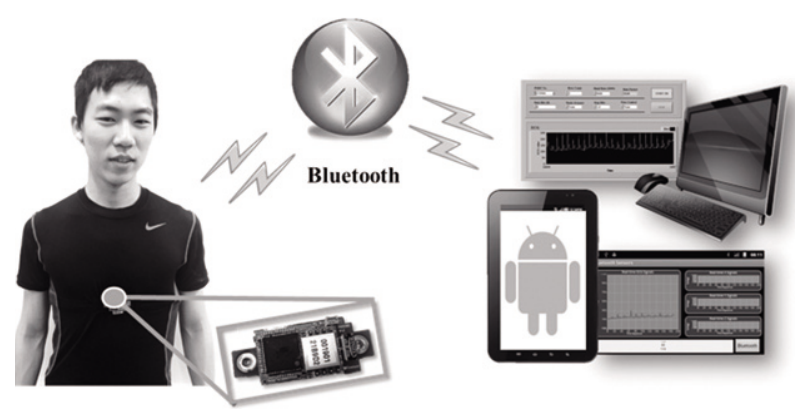

Fig. 1. Architecture of the mobile healthcare system.

\subsection{Hardware}

Fig. 2 shows the hardware part of the proposed Mhealthcare system. Hardware consists of sensor part(ECG module), data process part(MCU, ATmega128), and output part(smart device). ECG sensor is designed to be located at central part of chest in wearable shirts to increase the convenience of ECG measurement in daily life of the wearer.

ECG module which can measure ECG signal consists of LPF(Low Pass Filter) circuit, HPF(High Pass Filter) circuit and amplifier circuit. This circuit measures micro current signal from heart. The MCU(8 bits microprocessor of 16 $\mathrm{MHz}$ frequency) is responsible for data processing using UART, ADC, Timer/Count functions. The best methods of communication are Bluetooth that can communicate well with smart device. Nowadays most of mobile device such as cellular phone, smart phone, tablet PC has Bluetooth receiver inside. Android application is designed to monitor ECG wave like signal on the monitor. 
ECG sensor module and MCU are combined to a single battery. Combined board is supplied $3.7 \mathrm{~V}(500 \mathrm{mAh})$ from the battery.

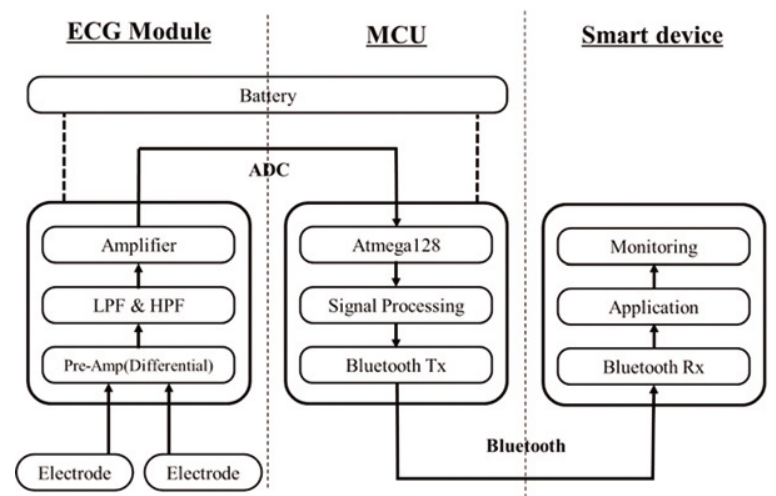

Fig. 2. Conceptional diagram of ECG monitoring System.

\subsubsection{Wearable shirt}

Fig. 3 shows the designed wearable shirt. Sensing units include tiny, wearable Bluetooth enable devices, such as body sensor node or chest belt, which aggregate and transmit collected vital signs to a smart monitoring device. Thus, the major communication protocol in this system is the Bluetooth wireless network. Indeed, the non-intrusive ECG sensor is necessary to be placed on the patient chest to measure the ECG signals through the body skin. In the design of the wearable shirt, the ECG sensor is attached on a chest belt via two conductive fabric electrodes with the size of $8 \mathrm{~cm}$.

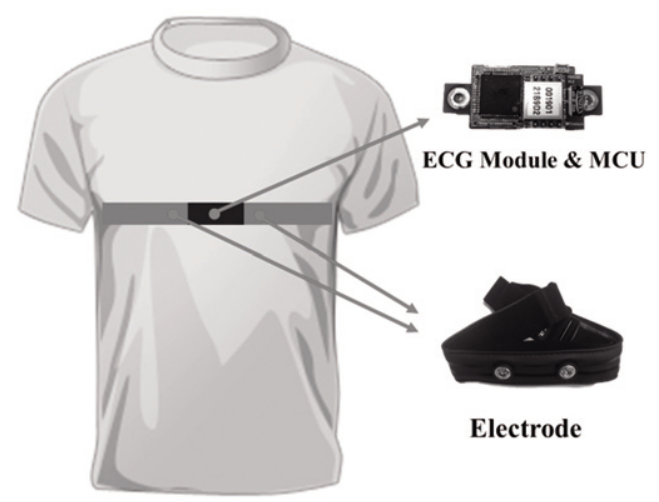

Fig. 3. ECG signal being measured wearable shirts designs.

The chest belt is then wrapped around the patient's inner chest part. In other means, the ECG chest belt is integrated into the wearable shirts to measure the ECG signals. The advantage of such design is that the tiny sensor is detachable and can be replaced without required to take off the entire suit. Moreover, the chest belt can hardly fall off due to sweat and has only little variation in frequencydependent impedance. Signals are least likely to be distorted during the measurement. Thus, the designed ECG sensor is essentially suitable for ECG measurement with just a simple attachment on the shirt.

The ECG module size is $2.2 \mathrm{~cm} \times 5.8 \mathrm{~cm}$ which has similar size to the size of a coin as illustrated in Fig. 4. This module which designed in laboratory measures the ECG based on general standard induction method which is the "lead 1" in 12-leads ECG. This method detects the potential differences between the right side and the left side of the heart. Basically, the electrode is placed on the right side of the leg to measure the ECG signals based on "lead 1" type 12-leads ECG, but the design ECG module can measure the ECG signals at other body part instead of only on the leg side.

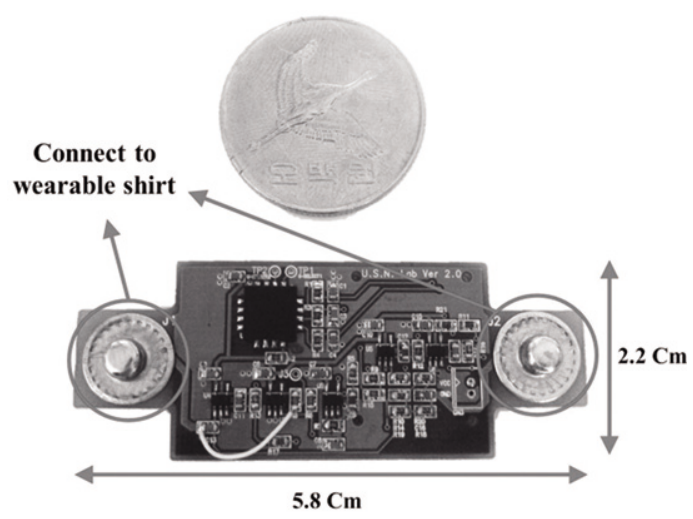

Fig. 4. The designed ECG module.

Table 1. Specification of the wearable ECG module

\begin{tabular}{cc}
\hline \hline Items & Specifications \\
\hline Electrodes & Strap (Polar) \\
Gain & $500(27 \mathrm{~dB})$ \\
Cutoff Frequency & $0.5 \mathrm{~Hz} \sim 34 \mathrm{~Hz}$ \\
Power & $3.3 \mathrm{~V}$ \\
\hline \hline
\end{tabular}

The chest belt electrodes performed signals measurement via analog signal conditioning circuit(ASCC) which is built with an amplifier, a low pass filter(LPF), and a high pass filter(HPF). The LPF and HPF are designed to remove noises resided in the analog signals. The signals are then transmitted to the smartphone device or PC through 
Bluetooth wireless communication. Fig. 5 depicts the progress flow of ECG measurement. The raw ECG signal is obtained from the ECG module and further processed by the analog signal conditioning circuit. In ASCC, the raw signal is filtered by a HPF with $0.34 \mathrm{~Hz}$ cut-off frequency and further amplified by active devices(OPA379, TI Inc., USA) for 27.6 times. After that, the similar process is repeated twice by the LPF with $23.4 \mathrm{~Hz}$ cut-off frequency and amplified by 10 times. The raw ECG signal is very weak, so it needs gradational amplification. The signals increased by several steps of filtering and amplifying are clearer than HPF passed signals. The ECG sensor is operated at $3.3 \mathrm{~V}$ and able to support several operating modes that consumed minimum of $1 \mu \mathrm{A}$ in sleep mode and maximum of $18 \mathrm{~mA}$ in active mode. Table 1 summarized the specification of the designed ECG sensor module.

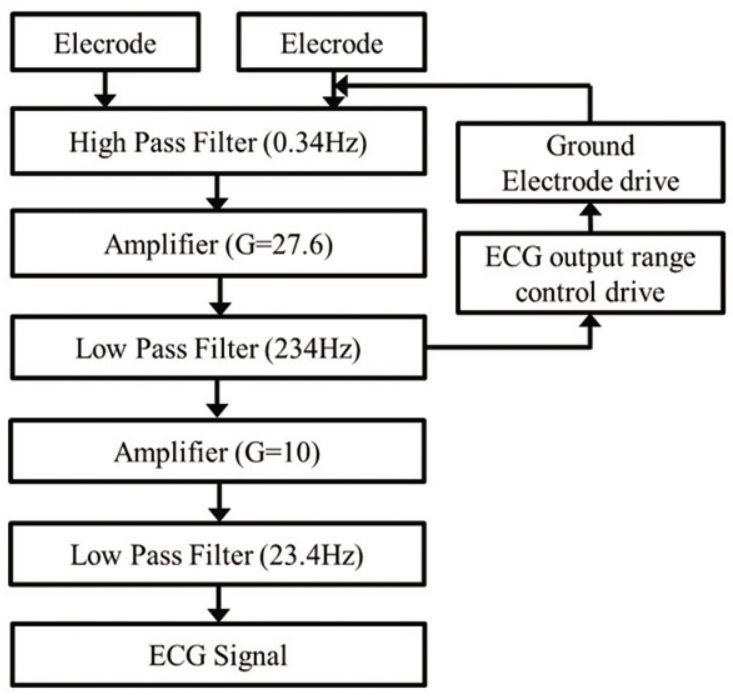

Fig. 5. Block diagram of ECG module interface.

\subsubsection{Microcontroller unit}

In the designed ECG sensor module, the microcontroller unit(MCU) board consists of an ATmega128(8 bit microcontroller unit of $16 \mathrm{MHz}$ ) microcontroller chip, Bluetooth and battery as illustrated in Fig. 6 Basically the microcontroller chip manipulated various functions including the $\mathrm{A} / \mathrm{D}$ signals conversion, timer count, and the I/O UART(Bluetooth) port. The ECG module sampling frequency is 8-bits count where an interrupt will be triggered at every specific count of 8-bits. The signal is further converted by $\mathrm{A} / \mathrm{D}$ conversion circuit for every interrupt occurred.

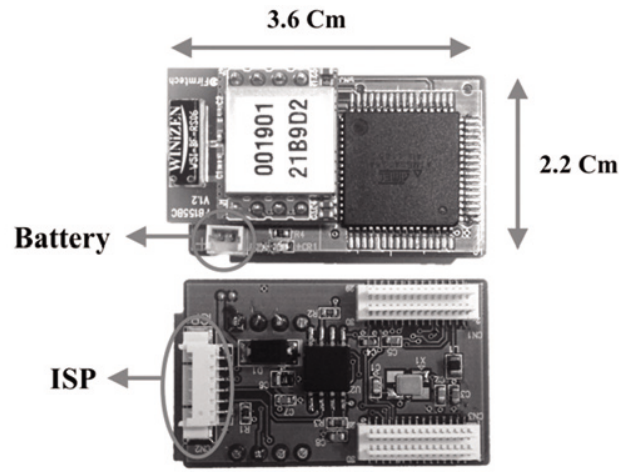

Fig. 6. The MCU board.

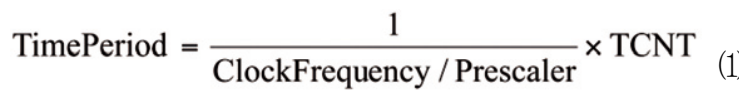

$$
\text { Sampling Frequency }=\frac{1}{\text { TimePeriod } \times n}
$$

The ECG signals have a sampling frequency of $100 \mathrm{~Hz}$. By referring to (1), the period for each interrupt time can be calculated and sampling frequency(refer to (2)) can be further computed based on the results obtained in (1). The variable input values of (1) are $16 \mathrm{MHz}$ for clock frequency, 128 for prescaler and 250 for TNCT. The time period obtained is $0.002 \mathrm{~s}$ which defined that the interrupt occurred for every $0.002 \mathrm{~s}$. In order to produce the 100 sampling frequency, the $\mathrm{A} / \mathrm{D}$ conversion should perform its task at every 5 interrupt intervals which is $0.01 \mathrm{~s}$. The total time for 100 sampling tasks took around $1 \mathrm{~s}$.

\subsubsection{Bluetooth}

Fig. 7 shows the selected Bluetooth module(FB155BC, Firmtech Co., Korea) which is able to communicate with the Bluetooth-enabled smartphone device once the pairing and connection are successful. The ATmega128 is connected to the Bluetooth via pin 7 (TXD) and pin 8 (RXD) where the signals can be transmitted using 9600 baud rate configuration.

The ATmega128 is required to send an operating command such as "at-btscan" for the Bluetooth device to start operating. The LED on the MCU will begin to blink if the Bluetooth module is ready to receive the data and the LED will be turn off if Bluetooth module is not applicable. Fig. 8 shows the location of LED on the board for Bluetooth status checking and Table 2 listed the Bluetooth module specification. 


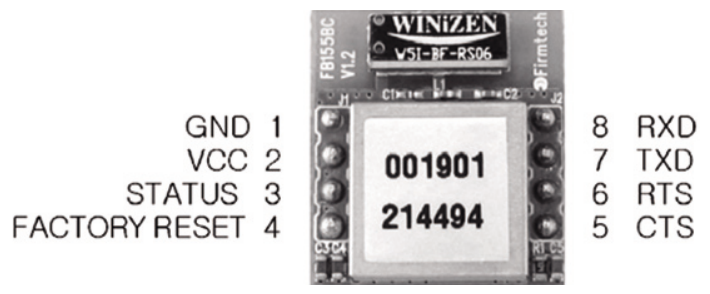

Fig. 7. FB155BC V1.2 Bluetooth module.

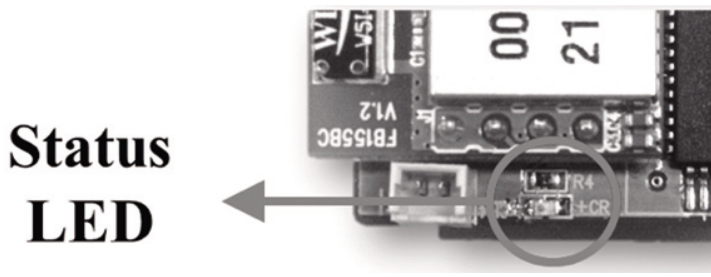

Fig. 8. Check Bluetooth status by LED.

Table 2. Specification of Bluetooth.

\begin{tabular}{ll}
\hline \hline \multicolumn{1}{c}{ Items } & \multicolumn{1}{c}{ Specifications } \\
\hline Version & V2.1 \\
Carrier Frequency & $2.4 \mathrm{GHz}$ ISM band \\
Supply Voltage & $3.3 \mathrm{VDC}+/-0.2 \mathrm{~V}$ \\
Power Class & Class $1(100 \mathrm{M})$ \\
Size & $18 \times 10 \mathrm{~mm}$ \\
\hline \hline
\end{tabular}

\subsubsection{Smartphone device}

An Android-based application is developed to receive the ECG signals via Bluetooth communication. The application is implemented in a development kit(HBESM5-S4210, Hanback Co., Korea) that runs on an operating system of Android version 2.3.5 version (GingerBread) and it is built upon the design of Galaxy Tab. This device supports various application developments and hardware controlling system to ease the developers in developing the application that suits their needs. Table 3 showed the specifications of HBE-SM5S4210 development kit.

\subsection{Software}

ECG signal was displayed on the Android-based smartphone device. This means that any user can measure their own ECG signals whenever they have want to if they have a smartphone device[7]. Only simple and basic
Table 3. Specification of HBE-SM5-S4210.

\begin{tabular}{cc}
\hline \hline Items & Specifications \\
\hline Processor & $\begin{array}{c}\text { Samsung Exnos 4210 } \\
\text { (ARM Cortex-A9 Dual Core) }\end{array}$ \\
Memory & 1 GByte LPDDR2 \\
Bluetooth & 1EA Bluetooth Ver 2.1 EDR Module \\
LCD & 7" TFT LCD $(800 \times 480)$ \\
Battery & Lithium Polymer Battery $(3.7 \mathrm{~V} / 3150 \mathrm{mAh})$ \\
Sensor & $\begin{array}{c}\text { Tri-axial Acceleration / Gyroscope Sensor } \\
\text { Magnetic Field Sensor/ Proximity Sensor }\end{array}$ \\
\hline \hline
\end{tabular}

procedure is required for the Bluetooth connection with the ECG sensors.

Fig. 9 shows the screenshot of the application`s respective functions. Basically, the graph will display user ECG signals while user status is shown on the right side of the graph. The application can evaluate the user's heart rate based on ECG signals. A notification of "normal" is shown if the user's heartbeat is in normal condition or else "abnormal" will be shown instead, which is illustrated in Fig. 9 The application also allowed user to scan, select, and connect to the ECG sensor by clicking on the "Bluetooth" option at the right bottom of the screen.

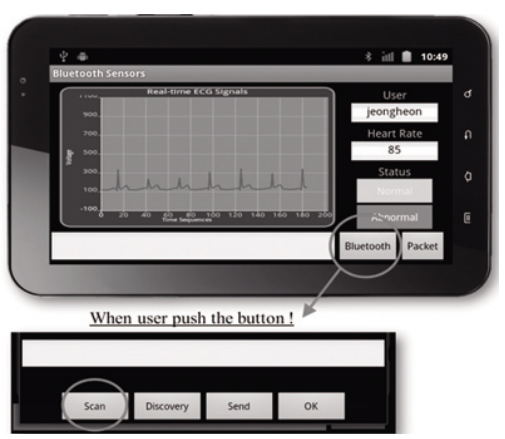

Fig. 9. Application configuration.

The foremost part of the application is the evaluation of the patient's status based on the heartbeat activities that are extracted from the received ECG signals as illustrated in Fig. 10[8]. If signal's width is ranged from $60 \mathrm{~ms}$ to $100 \mathrm{~ms}$ and R-to-R interval is between $0.8 \mathrm{~s}$ to $0.9 \mathrm{~s}$, it is considered as normal stage and vice versa[9]. Once the abnormal stage is reached, the warning and evaluation results will be displayed on the device screen for patient's review. The application will display a text of "Normal" in green color for normal stage while "Abnormal" in red color for every evaluation process. 


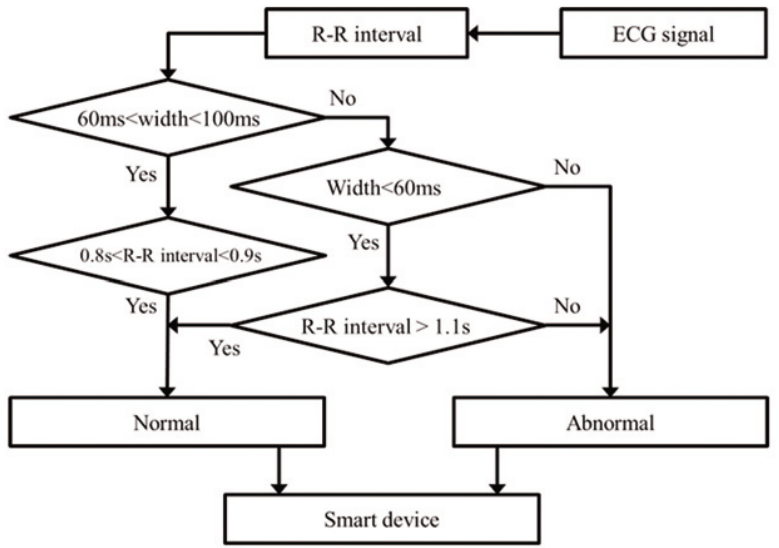

Fig. 10. Health status evaluation algorithm.

\section{EXPERIMENTAL METHODS AND RESULT}

\subsection{Experimental methods}

Experiments are conducted at the laboratory and nearby areas to validate the flexibility and reliability of the designed ECG wearable shirt. Fig. 11 described the experiment scenarios that are conducted. The participated testers performed four different actions including stand up and sit down(Fig. 11(a)), walking(Fig. 11(b)), climbing up and down the stairs(Fig. 11(c)), as well as run in place(Fig. 11(d)) by wearing the designed wearable shirt during the experiments. Those testers are able to view their own ECG signals in real-time on the device.

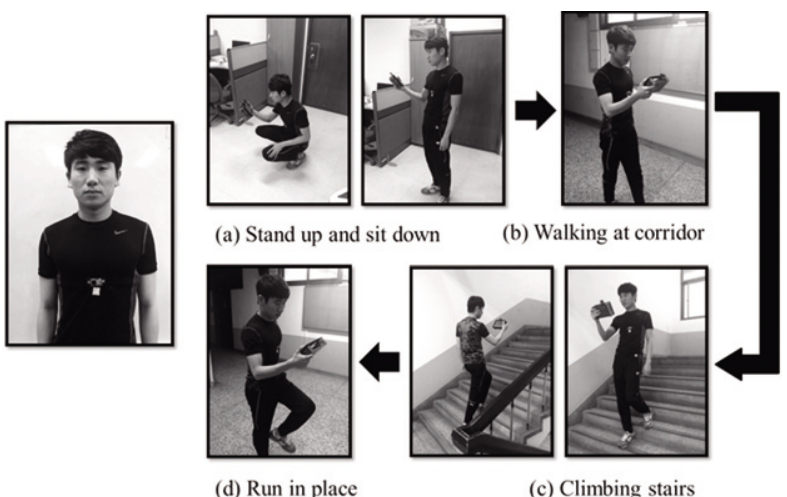

Fig. 11. The experiments for other actions.

\subsection{Experimental result}

The ECG signals received by performing the above mentioned actions are recorded for further evaluation. The following subsection illustrates the variation in ECG signal in different scenarios.

\subsubsection{Sitting down and standing up}

Fig. 12 shows signal distortion during the sit down and stand up motions. The signal distortion is denoted in a white square box in which the Fig. showed the signal distortion in sit down(top) and stand up(bottom) scenario respectively.

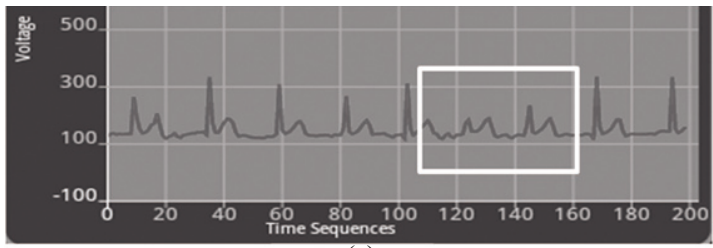

(a)

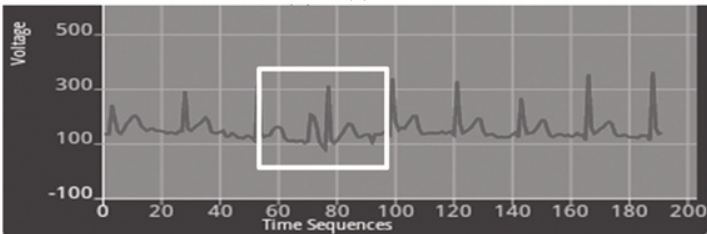

(b)

Fig. 12. ECG signal when standing up (a) and sitting down (b).

\subsubsection{Walking on corridor}

The ECG signals are not affected by the walking scenarios as shown in Fig. 13. It illustrated that the walking motion artifact can be easily removed by the ECG sensor module itself.

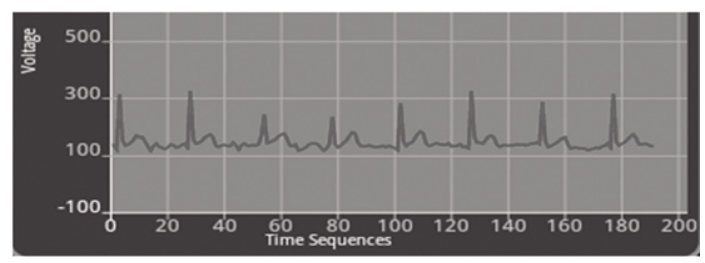

Fig. 13. ECG signal on walking.

\subsubsection{Climbing stairs}

Similar to the first scenarios, there are signal distortion occurred in the climbing stairs scenarios as well. Fig. 14(a) illustrated the signal distortion during climbing up motions and Fig. 14(b) showed the distortion during climbing down motion. 


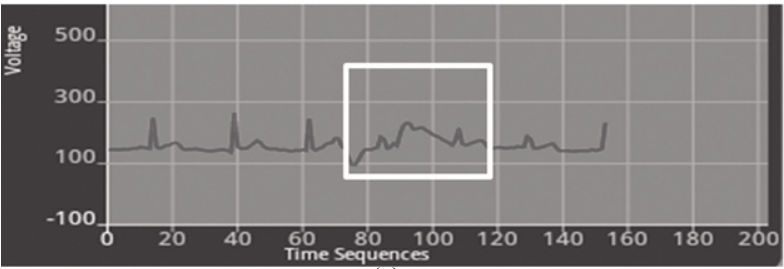

(a)

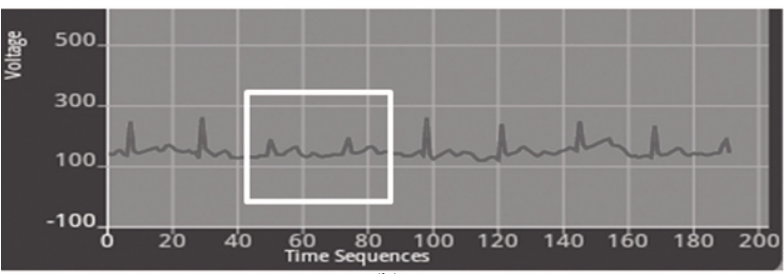

(b)

Fig. 14. ECG signal at climbing stairs ; going up stairs (a), going down stairs (b).

\subsubsection{Running in place}

Lastly, the sudden run in place scenarios generated the highest signal distortion among the four scenarios as illustrated in Fig. 15. However, once the running motion is stopped, the measured ECG signals were shown to be nondistorted signals.

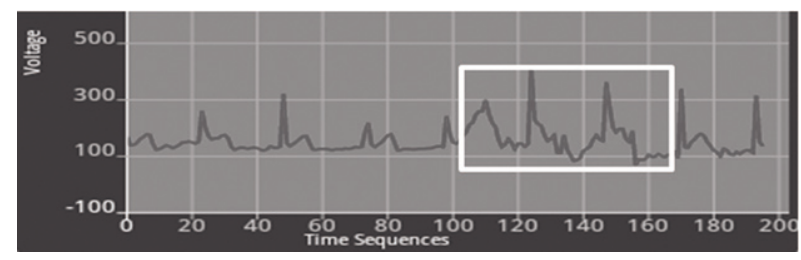

Fig. 15. ECG signal using running in place.

\section{CONCLUSIONS}

A mobile healthcare system was designed and developed to identify a person healthcare status in a more efficient and practical ways. The healthcare status is evaluated based on ECG signals and displayed on a smartphone device where no extra monitoring device is required. An ECG sensor module is integrated on the wearable shirt in which the ECG signal can be measured and transmitted to the smartphone device via Bluetooth wireless communication. An Android-based system is used to evaluate the received ECG signals and further classify a person status into either normal or abnormal state. The results revealed the reliability of ECG signals measurement under discrete scenarios with least noises and distortion occurred.

\section{ACKNOWLEDGMENT}

This research was financially supported by the Ministry of Education, Science Technology(MEST) and National Research Foundation of Korea(NRF) through the Human Resource Training Project for Regional Innovation.

\section{REFERENCES}

[1] W. Y. Chung, S. Bhardwaj, A. Purwar, D. S. Lee, and R. Myllyla, "A fusion health monitoring using ECG and accelerometer sensors for elderly persons at home", Proceedings of the 29th Annual International Conference of the IEEE EMBS, pp. 3818-3821, 2007.

[2] T. Takalokastari, S. J. Jung, D. D. Lee, and W. Y. Chung, "Real time drowsiness detection by WSN based wearable ECG measurement system", Journal of Sensor Science and Technology, vol. 20, no. 6, pp. 382387, 2011.

[3] S. J. Jung, Y. S. Seo, and W. Y. Chung, "Pulse wave analysis system using wrist type oximeter for u-Health service” J. Kor. Sensors Soc., vol. 19, no. 1, pp. 17-24, 2009.

[4] N. Sriraam, "An ubiquitous healthcare system using a wearable shirt for a smart home-a pilot study", 2010 IEEE EMBS(Biomedical Engineering and Sciences) Conference on, pp. 205-209, 2010.

[5] G. Bames, "A wearable, Bluetooth-enabled system for home health care", 24th Annual Conference and the Annual Fall Meeting of the Biomedical Engineering Society EMBS/BMES Conference, pp. 1879-1880, vol. 3, 2002.

[6] T. Wu, J. Hu, C. Gao, J. Zhao, and W. Ye, "Wireless monitoring system based on Bluetooth smart phones", International Conference on Net-working and Digital Society, pp. 648-651, 2010.

[7] A. Lymberis, "Advanced wearable health systems and applications - Research and development efforts in the european union", Engineering in Medicine and Biology Magazine IEEE, vol. 26, no. 3, pp. 29-33, 2007.

[8] S. C. Lee and W. Y. Chung, "A research for removing ECG noise and transmitting 1-channel of 3-axis accelerometer signal in wearable sensor node based on WSN", Journal of Sensor Science and Technology, vol. 20, no. 2, pp.137-144, 2011.

[9] J. Pan and W. J. Tompkins, "A real-time QRS detection algorithm”, IEEE Trans. Biomed Eng., vol. 32, no. 3, 
pp. 230-236, 1985.

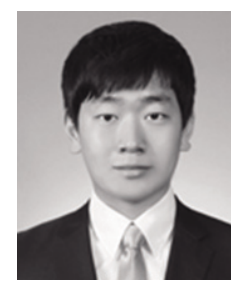

Jeong-Heon Kim was born in Korea. He received the B.S. degree in division of electronic and telecommunication engineering from Pukyong National University, Busan, Korea, He is now working towards the M.S. degree at the Ubiquitous Sensor Network Laboratory, Department of Electronic Engineering, Graduate School, Pukyong National University, Busan, Korea. His research interests include ubiquitous healthcare, embedded systems, and microcontroller.

Boon-Giin Lee was born in Malaysia. He received the B.S. degree in information technology majoring in software engineering from Multimedia University, Melaka, Malaysia, in 2007 and the M.S. degree majoring in ubiquitous information technology from Dongseo University, Busan, Korea, in 2009. He is now working towards the $\mathrm{Ph} . \mathrm{D}$. degree at the Ubiquitous Sensor Network Laboratory, Department of Electronic Engineering, Graduate School, Pukyong National University, Busan, Korea.
Seung-Chul Lee was born in Korea. $\mathrm{He}$ received his $\mathrm{Ph}$. Dgree in division of Electronic Enginnering from Pukyong National University, Busan, Korea. He is now working on KETI, Gyeonggido, Korea.

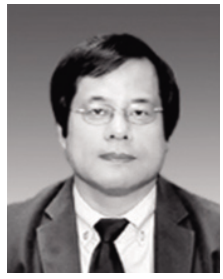

Wan-Young Chung was born in Korea. He received the B.S. and M.S. degrees in electronic engineering from Kyungpook National University, Daegu, Korea, in 1987 and 1989, respectively, and the Ph.D. degree in sensor engineering from Kyushu University, Fukuoka, Japan, in 1998. He received his third Doctor degree (Dr. of Science in Technology) from The University of Oulu, Finland, in 2009. He has been a Professor with the Department of Electronic Engineering, Pukyong National University, Korea, since September 2008. From 1999 to 2008, he was an Associate Professor at Dongseo University, Korea. His areas of interest include wireless sensor network, ubiquitous healthcare and automobile application, smart LED lighting with visible light communication and embedded systems. 